\title{
Assessment of female sex as a risk factor in atrial fibrillation in Sweden: nationwide retrospective cohort study

\author{
(c) (1) (9) OPEN ACCESS
}

\author{
Leif Friberg senior consultant cardiologist ${ }^{1}$, Lina Benson statistician ${ }^{2}$, Mårten Rosenqvist professor ${ }^{3}$, \\ Gregory Y H Lip professor ${ }^{4}$
}

${ }^{1}$ Karolinska Institute, Danderyd Hospital, Stockholm, Sweden; ${ }^{2}$ Karolinska Institute, Department of Clinical Science and Education, Södersjukhuset, Stockholm, ; ${ }^{3}$ Karolinska Institute, Department of Clinical Science and Education, Cardiology Unit, Södersjukhuset; ${ }^{4}$ University of Birmingham Centre for Cardiovascular Sciences, City Hospital, Birmingham, UK

\begin{abstract}
Objective To determine whether women with atrial fibrillation have a higher risk of stroke than men.

Design Nationwide retrospective cohort study.

Setting Patients with a diagnosis of atrial fibrillation in the Swedish hospital discharge register between 1 July 2005 and 31 December 2008. Information about drug treatment taken from the Swedish drug register.

Participants 100802 patients with atrial fibrillation at any Swedish hospital or hospital affiliated outpatient clinic with a total follow-up of 139 504 years at risk (median 1.2 years). We excluded patients with warfarin at baseline, mitral stenosis, previous valvular surgery, or who died within 14 days from baseline.
\end{abstract}

Main outcome measure Incidence of ischaemic stroke.

Results Ischaemic strokes were more common in women than in men (6.2\% v 4.2\% per year, $\mathrm{P}<0.0001)$. The univariable hazard ratio for women compared with men was 1.47 (95\% confidence 1.40 to 1.54), indicating a $47 \%$ higher incidence of ischaemic stroke in women than in men. Stratification according to the $\mathrm{CHADS}_{2}$ scheme showed increased stroke rates for women in all strata. After multivariable adjustment for 35 cofactors for stroke, an increased risk of stroke in women remained $(1.18,1.12$ to 1.24). Among patients with "lone atrial fibrillation" (age $<65$ years and no vascular disease), the annual stroke rate tended to be higher in women than in men, although this difference was not significant $(0.7 \% v 0.5 \%, \mathrm{P}=0.09)$. When low risk patients with $\mathrm{CHADS}_{2}$ scores of $0-1$ were stratified according to their $\mathrm{CHA}_{2} \mathrm{DS}_{2}$-VASc scores, women did not have higher stroke incidence than men at $\mathrm{CHA}_{2} \mathrm{DS}_{2}-\mathrm{VASc}$ scores of 2 or less.

Conclusion Women with atrial fibrillation have a moderately increased risk of stroke compared with men, and thus, female sex should be considered when making decisions about anticoagulation treatment.
However, women younger than 65 years and without other risk factors have a low risk for stroke, and do not need anticoagulant treatment.

\section{Introduction}

Several studies have indicated that women with atrial fibrillation are at higher risk of ischaemic stroke than men, ${ }^{1-6}$ although other studies have found no difference. ${ }^{378}$ The systematic review of stroke risk factors by the Stroke in Atrial Fibrillation Working Group concluded that female sex multiplied the risk of stroke by 1.6 -fold, and female sex has been incorporated into stroke risk assessment guidelines. ${ }^{9}{ }^{10}$

Differences in stroke risk between the sexes is of clinical relevance, and of great practical importance if few or no other stroke risk factors exist, and a decision regarding anticoagulation treatment is needed. It is also important to determine whether the generally recognised risk factors for stroke are equally important for men and women - that is, whether one common scheme for stroke risk stratification can be used for men and women with atrial fibrillation, or each sex should have different schemes.

The recent European Society of Cardiology guidelines recommend the use of the $\mathrm{CHA}_{2} \mathrm{DS}_{2}$-VASc score to refine stroke risk stratification, and female sex carries one point on this score. ${ }^{10}$ The guidelines clearly recommend that antithrombotic treatment is necessary in all patients with atrial fibrillation unless they are "age $<65$ and low risk." However, this point is still commonly misinterpreted for women with atrial fibrillation who are younger than 65 years and do not have any other risk factors (that is, they still have a $\mathrm{CHA}_{2} \mathrm{DS}_{2}$-VASc score of 1), in that they should be treated with "either oral anticoagulants or aspirin 75-325 mg daily, with oral anticoagulants preferred rather than 
aspirin." Indeed, should doctors recommend a physically active woman aged, for example, 45 years with atrial fibrillation (and no other risk factors) to take oral anticoagulants to avoid stroke, while recommend a man under the same circumstances to have no antithrombotic treatment? Furthermore, there remains uncertainty about whether the term "lone atrial fibrillation" is relevant in women, since their sex is considered a stroke risk factor. At a pragmatic level, young women with atrial fibrillation with female sex as the only risk factor $\left(\mathrm{CHA}_{2} \mathrm{DS}_{2}-\mathrm{VASc}\right.$ score 1) should not need anticoagulation treatment, if they fulfil the criteria of "age $<65$ and lone atrial fibrillation." 10

Unfortunately, the studies on which we base these decisions do not have much to say about such management situations, although they are commonly encountered in clinical practice. In a recent systematic review of stroke risk factors in atrial fibrillation, as part of the guidelines from the UK National Institute for Health and Clinical Excellence (NICE), ${ }^{3}$ five studies showed higher stroke risk in women than in men, whereas six other studies showed no difference in risk. The studies that did not find an excess risk for women were generally smaller than the studies that found increased risks for women and thus, the negative studies could have been underpowered to show a difference. The largest of these studies was the anticoagulation and risk factors in atrial fibrillation (ATRIA) cohort study ${ }^{1}$ of 13599 patients, which showed a higher risk for ischaemic stroke in women than in men (hazard ratio $1.5,95 \%$ confidence interval 1.2 to 1.8$)$. Furthermore, the NICE guidelines concluded that although female sex could be a stroke risk factor, there was no clear biological plausibility for this; therefore, female sex was not included in NICE's assessment algorithm for stroke risk. ${ }^{11}$ In a nationwide retrospective cohort study, we aimed to determine whether women with atrial fibrillation have a higher risk of stroke than men with atrial fibrillation.

\section{Methods}

We used the Swedish national discharge register to identify all individuals with a diagnosis for atrial fibrillation using code I489 of the ICD-10 (international classification of diseases, 10th revision; with or without subcodes A-F) between 1 July 2005 and 31 December 2008. This register contains records of all hospital admissions and all visits to hospital outpatient clinics for all patients with a Swedish civic registration number since 1987.

In the register, each hospital contact is recorded with one principal diagnosis and up to nine ancillary diagnoses, and up to 13 codes for surgical and other procedures. We used the register to obtain information about comorbidities as well as about events during follow-up. For current and previous diseases, we considered admissions from 1997 onwards (that is, from the year ICD-10 was implemented in Sweden). The web appendix defines the ICD-10 codes used to define endpoints and comorbidities (table w1).

We used both principal and secondary diagnoses, and the web appendix specifies the codes used for disease definitions. The register does not include information about principal diagnoses in $0.5-0.9 \%$ of admissions in somatic care. ${ }^{12}$ A diagnosis of atrial fibrillation or flutter in the register has a positive predictive value of $97 \% .^{13}$ The register's validity is evaluated annually, and is considered to be well suited for epidemiological studies by the National Board of Health and Welfare. ${ }^{14}$

We calculated each patient's stroke risk score according to the $\mathrm{CHADS}_{2}$ scheme. This score gives two points for a previous stroke, transient ischaemic attack, or systemic emboli, and one point each for heart failure, hypertension, diabetes, and age of
75 years or older. ${ }^{15}$ We also calculated the newer $\mathrm{CHA}_{2} \mathrm{DS}_{2}$-VASc score that, in addition to the $\mathrm{CHADS}_{2}$ score, gives two points for age of 75 years or older, and one point each for age 65-74 years, vascular disease, and female sex. ${ }^{2}{ }^{10}$

The index date was when the patient was first diagnosed with atrial fibrillation after 1 July 2005 . For the registration of events during follow-up, we applied a "blanking period" of 14 days after index. We used this period because transfers between hospitals and clinics were common, and early reappearances of a diagnosis were often closely related to a preceding hospital period-for example, a new code for an event that had been registered at another clinic a few days earlier. Diagnoses given on the index date and up to two weeks after that date were considered as reflecting comorbidity, and were not counted as endpoints during follow-up. Exclusion criteria were use of warfarin at baseline, death in conjunction with the index generating hospital period, mitral stenosis, or history of valvular surgery.

We obtained drug information from Anatomical Therapeutic Chemical classification codes in the national prescribed drugs register. This register has automatically stored detailed information about every pharmacy prescription in Sweden since 1 July 2005; therefore, we regarded this register to be almost $100 \%$ complete. Drugs used at baseline were those that had been collected at a pharmacy within 90 days of the index date. The only registered oral anticoagulant in Sweden during the study period was warfarin, with phenprocoumon used as an alternative on special license for the very few patients who were intolerant to warfarin.

\section{Statistical analysis}

We presented baseline characteristics descriptively, and tested differences between men and women with the $t$ and $\chi^{2}$ test. Annual incidence of stroke was calculated as events per 100 years at risk, with results expressed as percentages, and tested with Fisher's exact test. We used the Taylor series method to calculate $95 \%$ confidence intervals. Population attributable risk (\%) was calculated as $\left(I_{\text {population }}-I_{\text {men }}\right) / I_{\text {population }} \times 100$, where $I$ is the yearly incidence of stroke (number of events/years at risk). We presented survival graphically using the Kaplan-Meier method, and analysed rates using univariable and several multivariable Cox regressions using the Efron method of handling ties. In the multivariable models, we included comorbidities and use of drugs that had known associations with stroke, bleeding, or mortality ( 35 cofactors in total; table $1 \Downarrow$ ). Censoring was done at the earlier date of death or end of follow-up (31 December 2008). Primary analysis was done by intention to treat, and only included baseline variables, but a Cox regression was also estimated, which included exposure to acetylsalicylic acid, clopidogrel, and warfarin as time dependent variables.

For acetylsalicyclic acid and clopidogrel, we used the prescribed dose and the quantity of purchased drugs to calculate the number of days each prescription would last, and measured the exposed period from the date of purchase. For warfarin, since dose varies both among individuals and over time, we estimated each purchase to last three months. We estimated this period because a regulation in Sweden requires physicians not to prescribe more drugs than necessary for three months at a time (doctors may prescribe four three-month periods on the same occasion, but the patient can not claim it all at once). We disregarded gaps of at least 90 days between drug periods, and considered the patient to be on continuous drug treatment.

Interactions between all variables and sex were tested for the multivariable model, and selected interactions were presented 
in a forest plot. Modelling interactions directly in the Cox model renders similar interpretation, as if separate Cox regressions had been performed on subgroups but with the additional advantage of testing for differences between subgroups. We investigated the scaled Schoenfeld residuals and "dfbetas" from the primary model for violations to the proportional hazards assumption and possible outliers, respectively, but detected no problems. The linear association between age and outcome was investigated for the Martingale residuals and found to be acceptable, but for the sensitivity analysis, age was also modelled using restricted cubic splines.

We considered $\mathrm{P}<0.05$ to be significant. Analyses were performed in R version 2.14.2 (R Foundation for Statistical Computing) and OpenEpi 2.3.1 (www.openepi.com/OE2.3/ Menu/OpenEpiMenu.htm).

\section{Results}

We identified 182678 unique people who had been diagnosed with atrial fibrillation on one or several occasions during the 3.5 years we studied. In total, these represented 1845906 hospital contacts. We excluded 73591 patients who used warfarin (or in a few cases, phenprocoumon) at index, 7078 who died in conjunction with the index hospital period, 1283 who had a diagnosis of mitral stenosis, and 7639 who had undergone valvular surgery previously. Some patients were excluded because of more than one criterion (web appendix, table w2). After exclusions, 100802 patients remained in the study (table 1).

During a median follow-up of 1.2 years (range 0-3.5, total 139 504 years at risk), we identified 7221 patients who had a thromboembolic stroke (annual stroke rate 5.2\%, 95\% confidence interval $5.1 \%$ to $5.3 \%$ ). Ischaemic strokes were more common in women than in men (4264 strokes during 69005 years at risk in women, and 2957 strokes during 70594 years at risk in men, corresponding to annual stroke rates of $6.2 \%$ and $4.2 \%$ respectively, $\mathrm{P}<0.0001$ ), and women with atrial fibrillation had a $47 \%$ higher incidence of ischaemic stroke than men with atrial fibrillation (univariable hazard ratio $1.47,95 \%$ confidence interval 1.40 to 1.54 ). Visually, the difference in unadjusted stroke incidence between men and women was larger in the highest age groups (women $v$ men: age $<65$ years, hazard ratio 1.09 (95\% confidence interval 0.86 to $1.39, \mathrm{P}=0.48$ ); $65-74$ years, 1.04 ( 0.91 to $1.20, \mathrm{P}=0.53$ ); $\geq 75$ years, 1.24 ( 1.18 to 1.30 , $\mathrm{P}<0.0001$ ); fig $1 \Downarrow)$.

The population attributable risk of female sex on stroke was $19.0 \%$ (95\% confidence interval $17.0 \%$ to $20.1 \%$ ) - that is, patients would have had $19 \%$ fewer strokes if they were all male. However, this univariable analysis did not adjust for an increase of six years of mean age in women, compared with men (81 $v 75$ years). Women also had more risk factors for stroke than men (table 1). Women had a history of previous stroke, dementia, frequent falls, and hypertension more often, and men had a history of myocardial infarction, diabetes, liver and renal problems, and alcohol abuse more often. Women scored higher than men on the $\mathrm{CHADS}_{2}$ score (mean $2.3 v 1.9$; $\mathrm{P}<0.0001$ ), which was partly due to the increased mean age in women (age $\geq 75$ years holds one point). When we stratified patients according to $\mathrm{CHADS}_{2}$ scores, women had a higher incidence of stroke than men in every group (fig $2 \Downarrow$ ).

When we stratified low risk patients with $\mathrm{CHADS}_{2}$ scores of 0-1 according to the $\mathrm{CHA}_{2} \mathrm{DS}_{2}-\mathrm{VASc}$ score (which gives one point for female sex), we found that women did not have higher stroke incidence than men at $\mathrm{CHA}_{2} \mathrm{DS}_{2}-\mathrm{VASc}$ scores of 2 or less (fig $3 \Downarrow$ ). Patients with combinations of the "new" risk factors (that is, those not included in $\mathrm{CHADS}_{2}$ ) did show stroke rates of about $4 \%$ annually, and thus were not really at low risk.

Among patients with lone atrial fibrillation, defined as $\mathrm{CHADS}_{2}$ score 0 and age $<65$ years, the annual stroke rate tended to be higher in women than in men but this difference was not significant $(0.7 \% v 0.5 \%, \mathrm{P}=0.09 ; 31$ strokes during 4626 years at risk in women, 53 strokes during 11677 years in men).

Drug treatments differed between the sexes. Because we excluded patients who used warfarin at baseline, this excluded group included more men than women. Warfarin was used by 30364 (36\%) women at index compared with 43227 (44\%) of men. Women used $\beta$ blockers and digoxin more often (rate control drugs), whereas men used class I or class III anti-arrhythmic drugs more often (rhythm control drugs).

After adjustment for differences in background characteristics, an association remained between female sex and increased risk of ischaemic stroke (hazard ratio 1.18, 95\% confidence interval 1.12 to 1.24$)$. We found similar results when we included acetylsalicylic acid, clopidogrel, and warfarin as time dependent covariates $(1.14,1.09$ to 1.19 ; table $2 \Downarrow)$. Web appendix table w3 provides all risk estimates of the different covariates included in the Cox model.

We found significant interactions with sex for age as a continuous variable, history of stroke, vascular disease, and use of diuretics. However, in all patients, except for narrowly defined ischaemic stroke, the hazard ratio of ischaemic stroke for sex remained above 1 (women $v$ men), although not significantly in some patients. The interaction with $\mathrm{CHADS}_{2}$ score was also significant, in which the difference in risk of ischaemic stroke between the sexes seemed to diminish in the presence of more comorbidities (fig $4 \Downarrow$ ).

\section{Discussion}

In this large cohort study of more than 100000 patients with atrial fibrillation without warfarin treatment, unadjusted incidence of thromboembolic stroke was $47 \%$ higher in women than in men. Although women had more risk factors for stroke than men, they still had an $18 \%$ higher risk of stroke than men even after adjustment.

In the Atrial Fibrillation Investigators' meta-analysis of five randomised trials in 1990, researchers found a $20 \%$ higher risk in women than in men, although this analysis was not adjusted for cofactors. ${ }^{8}$ Larger differences in stroke risk between the sexes were found in the Framingham study (hazard ratio 1.9), Euro heart survey (1.8), and ATRIA study (1.5), ${ }^{14} 16{ }^{17}$ but these studies were substantially smaller than the present study. Indeed, our study comprised over 7000 events of ischaemic stroke, whereas the largest study investigating sex differences previously, the ATRIA study, only had 369 events; none of the other studies exceeded 100 events. Furthermore, our study adjusted for 35 cofactors whereas the ATRIA and Framingham studies only adjusted for seven and five cofactors, respectively. The difference in risk between men and women was not the same in all subgroups. At any $\mathrm{CHADS}_{2}$ score, the point estimate of the relative stroke risk was higher in women than in men (fig 2). In multivariable analysis, we saw significant differences in stroke risk in patients aged 75 years and older, and those with $\mathrm{CHADS}_{2}$ scores lower than 4 .

Sex had a significant interaction with the history of stroke. Women with ischaemic stroke as the primary diagnosis had the same stroke risk as men. A possible reason could be related to the finding of a general increased threshold for warfarin treatment in women. If women, after having a stroke, received 
intense treatment similar to that given to men, the difference in stroke rate between the sexes would probably be reduced. A similar tendency was found in relation to a history of myocardial infarction. Such events could result in more standardised care that ensures women receive the same level of care as men. However, our multivariable analysis with exposure to warfarin, acetylsalicylic acid, and clopidogrel used as time dependent covariates only reduced the estimated hazard ratio by a small degree (from 1.18 to 1.14 ). Therefore, differences in medical care probably had a marginal role in the observed differences in stroke risk.

The absolute risk of atrial fibrillation related stroke in young and otherwise healthy women (that is, aged $<65$ years and with lone atrial fibrillation) was low and only non-significantly higher than in men $(0.7 \% v 0.5 \%, \mathrm{P}=0.09)$, indicating that female sex in the absence of other risk factors for stroke would not indicate a need for antithrombotic treatment. Nevertheless, healthy women aged 65-74 years old had an annual stroke rate of 1.9\%, which may merit anticoagulation and evaluation of subtler risks such as absolute levels of blood pressure, whether the patient is aged closer to 65 years or to 75 years, and consideration of other risk factors beyond those in the risk stratification schemes in order to decide on optimal treatment. ${ }^{18}$ Eckman and colleagues ${ }^{19}$ suggested that treatment with new and "safer" oral anticoagulant drugs could be an option even at this low absolute risk, for which the threshold for treatment might be an annual stroke rate of $0.9 \%$. This observation is pertinent since aspirin is less effective than new oral anticoagulant drugs for stroke prevention and has a similar risk for major bleeding (and intracranial haemorrhage) ${ }^{20}$ and the evidence supporting aspirin use for stroke prevention in atrial fibrillation is weak. ${ }^{21}$

The European Society of Cardiology's guidelines ${ }^{10}$ recommend antithrombotic treatment for stroke prevention in all patients with atrial fibrillation unless they are low risk (that is, age $<65$ years and with lone atrial fibrillation); therefore, young women who have no risk factors would fall into this category, and the present study supports this. Thus, patients with "female sex" as the only risk factor (but who would still have a $\mathrm{CHA}_{2} \mathrm{DS}_{2}-\mathrm{VASc}$ score of 1 on that basis) would not need anticoagulation treatment, if they fulfil the criteria of being younger than 65 years and have lone atrial fibrillation..$^{18}$ The central question is to carefully define patients who are truly at low risk of atrial fibrillation related stroke, which the $\mathrm{CHA}_{2} \mathrm{DS}_{2}$-VASc score can help with, as shown by performing best in identifying such patients. $^{18}$

Many risk factors have continuous range of risk, even though they are treated dichotomously in risk assessment schemes. For example, degrees of hypertension, diabetes, heart failure, and vascular disease exist, and the disease (or risk factor) burden is relevant. This is particularly evident with age, by starting to rise from age 65 years onwards, ${ }^{22}$ and rises by 1.5 -fold per decade. ${ }^{9}$ In some situations, there are suspicions about underlying or incipient disease that could influence stroke risk, but yet without definite diagnosis. Indeed, assessments of patient risk should integrate various factors, such as bleeding risk and patient values and preferences, to reach an informed decision about the appropriate treatment. ${ }^{23}$ In borderline situations, in which a decision about whether to give anticoagulation treatment weighs in the balance, we suggest that female sex should probably tip the scale towards initiating treatment.

\section{Limitations}

We had no information in the registers about patients with atrial fibrillation who were managed exclusively in primary care or private care, nor did we have any information about those with undiagnosed, silent paroxysmal atrial fibrillation. However, this limitation is intrinsic to all similar studies, including those used to validate the $\mathrm{CHADS}_{2}$ score. ${ }^{15}$ The ATRIA study, which comprised 17974 selected individuals, only reported a prevalence in the general population of $0.95 \%$. Our nationwide cohort study, comprising more than ten times the number of patients, found that atrial fibrillation had a prevalence of $2 \%$ of the Swedish population. Although undercoding of atrial fibrillation in the register might still be possible, it is probably much less so in our study, compared with any other major study of atrial fibrillation done previously that we are aware of.

The patients in the study were derived from a Swedish hospital based cohort. Although patients could alternate between hospital and primary care, it might be assumed that patients with atrial fibrillation who go to hospital are sicker, in one way or the other, than patients who do not go to hospital. Thus, we do not know whether our findings applied to patients managed exclusively in primary care, or even to those with silent (sometimes as yet undiagnosed) atrial fibrillation. To some extent, these limitations are counterbalanced by the fact that our cohort study consecutively included all patients with atrial fibrillation on a nationwide scale for several years.

The Swedish hospital discharge register is subjected to annual quality control to ascertain completeness of information, and less than $1 \%$ of hospital periods lack a proper discharge record with at least one principal diagnosis as cause for the care. ${ }^{13} 14$ For some important diagnoses such as myocardial infarction and heart failure, external validation of the diagnostic accuracy is good. ${ }^{24}{ }^{25}$ For many other diagnoses, we know little about the validity of the register, and some comorbidities could have been under-reported (especially for hypertension), whereas over-reporting was probably much rarer. Thus, patients might have had more risk factors than we were aware of and could adjust for, but again, this limitation is inherent in all forms of registry studies.

In our study, women were older and had more risk factors for stroke than men, and thus, residual confounding (that we were unable to adjust for) might have been evident. Our only source of information was medical registers, with limited information about degree or severity of illness and about lifestyle factors such as obesity, smoking, and drinking that could also affect outcome. Also, our analysis did not account for echocardiographic data. Indeed, failure to adjust for underlying differences in cofactors could have given an exaggerated picture of differences between the sexes. Other studies, which adjusted for far fewer cofactors than ours, showed much larger differences in stroke risk between men and women than our study. ${ }^{17826}$ Also, observational studies have generally shown larger differences than randomised trials. ${ }^{1478172627}$ The most probable reason is that randomisation evens out some differences in background characteristics more efficiently than multivariable adjustments. The finding of a relatively small difference in risk between the sexes found in our study therefore could have been a result of our successful adjustment for many cofactors.

We selected patients for the study on the basis that they did not use warfarin at baseline. However, more men than women ( $44 \%$ $v 36 \%$ ) were using warfarin. A higher threshold for anticoagulation for women than for men could have resulted in selection bias. Women who did not receive warfarin, but perhaps would have had it if the sexes were treated equally, naturally carry an increased stroke risk. Thus, the consequence would have been an exaggeration of the risk differences between men and women. 


\section{What is already known on this topic}

Several studies have indicated that women with atrial fibrillation are at higher risk for ischaemic stroke than men, although other studies found no such difference

With few other risk factors to consider, female sex might be important in making decisions regarding anticoagulation treatment

\section{What this study adds}

Women with atrial fibrillation have a moderately increased risk of stroke compared with men with atrial fibrillation Women younger than 65 years and without other risk factors for stroke have the same low risk as men with the same characteristics, and do not need anticoagulant treatment

Contributors: LF had full access to all of the data in the study and takes responsibility for the integrity of the data and the accuracy of the data analysis. LF wrote the first manuscript draft and participated in revisions. LB participated in the statistical analysis and revision of the manuscript. MR and GYHL participated in drafting and revisions of the manuscript. Funding: The study was supported by the Swedish Heart and Lung Foundation, Stockholm County Council, and Board of Benevolence of the Swedish Order of Freemasons.

Competing interests: All authors have completed the Unified Competing Interest form at www.icmje.org/coi_disclosure.pdf (available on request from the corresponding author) and declare: the study received support from the Swedish Heart and Lung Foundation, Stockholm County Council and Board of Benevolence of the Swedish Order of Freemasons for the submitted work; LF is a consultant to Sanofi-Aventis,

Boehringer-Ingelheim, and Bristol-Myers Squibb; LB has nothing to declare; MR is a consultant to Sanofi-Aventis and Nycomed, Sweden, and has been national coordinator for the RECORD, REALISE, and ARISTOTLE study; GYHL has received funding for research, educational symposia, consultancy, and lecturing from different manufacturers of drugs used for the treatment of atrial fibrillation and thrombosis; no other relationships or activities that could appear to have influenced the submitted work.

Ethical approval: The study was approved by the ethical committee of Karolinska Institute (EPN 2008/433-32).

Data sharing: An appendix with additional tables is available online or directly from LF.

1 Fang MC, Singer DE, Chang Y, Hylek EM, Henault LE, Jensvold NG, et al. Gender differences in the risk of ischemic stroke and peripheral embolism in atrial fibrillation: the AnTicoagulation and Risk factors In Atrial fibrillation (ATRIA) study. Circulation 2005;112:1687-91.

2 Lip GY, Nieuwlaat R, Pisters R, Lane DA, Crijns HJ. Refining clinical risk stratification for predicting stroke and thromboembolism in atrial fibrillation using a novel risk factor-based approach: the Euro heart survey on atrial fibrillation. Chest 2010;137:263-72.

3 Hughes M, Lip GY. Stroke and thromboembolism in atrial fibrillation: a systematic review of stroke risk factors, risk stratification schema and cost effectiveness data. Thromb Haemost 2008;99:295-304.

4 Wang TJ, Massaro JM, Levy D, Vasan RS, Wolf PA, D'Agostino RB, et al. A risk score for predicting stroke or death in individuals with new-onset atrial fibrillation in the community: the Framingham Heart Study. JAMA 2003;290:1049-56.

5 Hart RG, Pearce LA, McBride R, Rothbart RM, Asinger RW. Factors associated with ischemic stroke during aspirin therapy in atrial fibrillation: analysis of 2012 participants in the SPAF I-III clinical trials. The Stroke Prevention in Atrial Fibrillation (SPAF) Investigators. Stroke 1999;30:1223-9.

6 Lane DA, Lip GY. Female gender is a risk factor for stroke and thromboembolism in atria fibrillation patients. Thromb Haemost 2009;101:802-5.

7 Humphries KH, Kerr CR, Connolly SJ, Klein G, Boone JA, Green M, et al. New-onset atrial fibrillation: sex differences in presentation, treatment, and outcome. Circulation 2001:103:2365-70.

8 Atrial Fibrillation Investigators. Risk factors for stroke and efficacy of antithrombotic therapy in atrial fibrillation. Analysis of pooled data from five randomized controlled trials. Arch Intern Med 1994;154:1449-57.
9 Stroke Risk in Atrial Fibrillation Working Group. Independent predictors of stroke in patients with atrial fibrillation: a systematic review. Neurology 2007;69:546-54.

10 Camm AJ, Kirchhof P, Lip GY, Schotten U, Savelieva I, Ernst S, et al. Guidelines for the management of atrial fibrillation: the task force for the management of atrial fibrillation of the European Society of Cardiology (ESC). Eur Heart J 2010;31:2369-429.

11 National Collaborating Centre for Chronic Conditions. Atrial fibrillation: national clinical guideline for management in primary and secondary care. Royal College of Physicians, 2006.

12 Ludvigsson JF, Andersson E, Ekbom A, Feychting M, Kim JL, Reuterwall C, et al. External review and validation of the Swedish national inpatient register. BMC Pub Health 2011;11:450

13 Smith JG, Platonov PG, Hedblad B, Engstrom G, Melander O. Atrial fibrillation in the Malmo Diet and Cancer study: a study of occurrence, risk factors and diagnostic validity. Eur J Epidemiol 2010;25:95-102

14 Kodningskvalitet i patientregistret- slutenvård 2008 , Socialstyrelsen [only available in Swedish; "Report on the validity of the National Hospital Discharge Register in Sweden"] National Board of Health and Welfare, 2010.

15 Gage BF, Waterman AD, Shannon W, Boechler M, Rich MW, Radford MJ. Validation of clinical classification schemes for predicting stroke: results from the National Registry of Atrial Fibrillation. JAMA 2001;285:2864-70.

16 Dagres N NR, Vardas PE, Andresen D, Lévy S, Cobbe S, Kremastinos D, et al. Gender-related differences in presentation, treatment, and outcome of patients with atrial fibrillation in Europe: a report from the Euro heart survey on atrial fibrillation. Am J Coll Cardiol 2007;49:572-7.

17 Singer DE, Chang Y, Fang MC, Borowsky LH, Pomernacki NK, Udaltsova N, et al. The net clinical benefit of warfarin anticoagulation in atrial fibrillation. Ann Intern Med 2009;151:297-305

18 Lip GY. Stroke in atrial fibrillation: epidemiology and thromboprophylaxis. J Thromb Haemost 2011;9(suppl 1):344-51.

19 Eckman MH, Singer DE, Rosand J, Greenberg SM. Moving the tipping point: the decision to anticoagulate patients with atrial fibrillation. Circ Cardiovasc Qual Outcomes 2011;4:14-21.

20 Connolly SJ, Eikelboom J, Joyner C, Diener HC, Hart R, Golitsyn S, et al. Apixaban in patients with atrial fibrillation. N Engl J Med 2011;364:806-17.

21 Lip GY. The role of aspirin for stroke prevention in atrial fibrillation. Nat Rev Cardio 2011;8:602-6.

22 van Walraven C, Hart RG, Connolly S, Austin PC, Mant J, Hobbs FD, et al. Effect of age on stroke prevention therapy in patients with atrial fibrillation: the atrial fibrillation investigators. Stroke 2009;40:1410-6

23 Lip GYH, Andreotti F, Fauchier L, Huber K, Hylek E, Knight E, et al. Bleeding risk assessment and management in atrial fibrillation patients: executive summary of a position document from the European Heart Rhythm Association, endorsed by the European Society of Cardiology Working Group on Thrombosis. Thromb Haemostat 2011, doi:10. 1160/TH11-10-0690.

24 Hammar N, Ahlbom A, Sandberg E, Alfredsson L. Time trends in the incidence of and mortality from acute myocardial infarction in Swedish women. Epidemiology 1996; 7:654-5.

25 Ingelsson E, Arnlov J, Sundstrom J, Lind L. The validity of a diagnosis of heart failure in a hospital discharge register. Eur J Heart Fail 2005;7:787-91.

26 Friberg J, Scharling H, Gadsboll N, Truelsen T, Jensen GB. Comparison of the impact of atrial fibrillation on the risk of stroke and cardiovascular death in women versus men (the Copenhagen City Heart Study). Am J Cardiol 2004;94:889-94.

27 Patients with nonvalvular atrial fibrillation at low risk of stroke during treatment with aspirin: Stroke Prevention in Atrial Fibrillation III Study. The SPAF III Writing Committee for the Stroke Prevention in Atrial Fibrillation Investigators. JAMA 1998;279:1273-7.

Accepted: 19 April 2012

\section{Cite this as: BMJ 2012;344:e3522}

This is an open-access article distributed under the terms of the Creative Commons Attribution Non-commercial License, which permits use, distribution, and reproduction in any medium, provided the original work is properly cited, the use is non commercial and is otherwise in compliance with the license. See: http://creativecommons.org/licenses/by$\mathrm{nc} / 2.0 /$ and http://creativecommons.org/licenses/by-nc/2.0/legalcode. 


\section{Tables}

\section{Table 1/ Baseline characteristics of study groups. Data are no (\%) of patients unless stated otherwise}

\begin{tabular}{|c|c|c|}
\hline & Men & Women \\
\hline No $(\%)$ & $50135(49.7)$ & $50667(50.3)$ \\
\hline \multicolumn{3}{|l|}{ Age (years) } \\
\hline Mean (standard deviation) & $74.7(13.5)$ & $80.9(10.6)$ \\
\hline Median & 78 & 83 \\
\hline \multicolumn{3}{|l|}{ History of atrial fibrillation } \\
\hline First ever diagnosis $<7$ days & $30905(62)$ & $31469(62)$ \\
\hline \multicolumn{3}{|l|}{ Medical history more common in women } \\
\hline Thyroid disease & $1218(2)$ & $5265(10)$ \\
\hline Frequent falls & $2990(6)$ & $6368(13)$ \\
\hline Dementia & $2444(5)$ & $3571(7)$ \\
\hline Pulmonary embolism & $784(2)$ & $1111(2)$ \\
\hline \multicolumn{3}{|l|}{ Thromboembolism } \\
\hline Ischaemic stroke ${ }^{*}$ & $6625(13)$ & $8095(16)$ \\
\hline Other thromboembolism† & $3130(6)$ & $3757(7)$ \\
\hline Anaemia & $5872(12)$ & $7169(14)$ \\
\hline Hypertension & $19188(38)$ & $23511(46)$ \\
\hline Heart failure & $15491(31)$ & $17644(35)$ \\
\hline \multicolumn{3}{|l|}{ Medical history more common in men, bleeding } \\
\hline Intracranial bleeding & $1314(3)$ & $1015(2)$ \\
\hline Other major bleeding & $3229(6)$ & $3231(6)$ \\
\hline Use of pacemaker & $3650(7)$ & $3272(6)$ \\
\hline Diabetes & $9272(18)$ & $8423(17)$ \\
\hline Emphysema or chronic obstructive pulmonary disease & $4393(9)$ & $3793(7)$ \\
\hline \multicolumn{3}{|l|}{ Medical history more common in men, vascular disease } \\
\hline Myocardial infarction & $11104(22)$ & $9224(18)$ \\
\hline Other vascular disease $\neq$ & $2699(5)$ & $2244(4)$ \\
\hline Cancer within three years & $7033(14)$ & $4385(9)$ \\
\hline Platelet defects or coagulopathy & $809(2)$ & $561(1)$ \\
\hline Renal disease & $3668(7)$ & $2331(5)$ \\
\hline Liver disease & $870(2)$ & $541(1)$ \\
\hline Alcohol index§ & $2715(5)$ & $544(1)$ \\
\hline \multicolumn{3}{|l|}{ Stroke risk score (mean (standard deviation)) } \\
\hline $\mathrm{CHADS}_{2}$ & $1.9(1.4)$ & $2.3(1.4)$ \\
\hline $\mathrm{CHA}_{2} \mathrm{DS}_{2}-\mathrm{VASc}$ & $3.0(1.8)$ & $4.4(1.7)$ \\
\hline \multicolumn{3}{|l|}{ Use of antithrombotic drugs } \\
\hline Acetylsalicylic acid only & $28874(58)$ & $32044(63)$ \\
\hline Clopidogrel only & $908(2)$ & $1060(2)$ \\
\hline Acetylsalicylic acid and clopidogrel & $3013(6)$ & $2063(4)$ \\
\hline None of the above & $17340(35)$ & $15500(31)$ \\
\hline \multicolumn{3}{|l|}{ Use of other drugs } \\
\hline$\beta$ blockers & $30016(60)$ & $33114(65)$ \\
\hline Class 1 anti-arrhythmic drugs & $1249(2)$ & $934(2)$ \\
\hline Class 3 anti-arrhythmic drugs & $4190(8)$ & $3257(6)$ \\
\hline Verapamil or diltiazem & $1862(4)$ & $2638(5)$ \\
\hline Calcium blocker dihydropyridine & $7869(16)$ & $8871(18)$ \\
\hline Digoxin & $10289(21)$ & $14640(29)$ \\
\hline
\end{tabular}




\section{Table 1 (continued)}

\begin{tabular}{lcc} 
& Men & Women \\
Diuretics & $24528(49)$ & $31898(63)$ \\
\hline $\begin{array}{l}\text { Angiotensin converting enzyme inhibitors and angiotensin } \\
\text { receptor blockers }\end{array}$ & $20687(41)$ & $19519(39)$ \\
\hline Statin & $13282(26)$ & $9717(19)$ \\
\hline
\end{tabular}

All comparisons between men and women were $P<0.05$, except for history of atrial fibrillation $(P=0.13)$.

*Narrow definition: previous diagnosis of ischaemic stroke (ICD-10 code I63) as principal diagnosis only.

†Narrow definition: previous secondary diagnosis of ischaemic stroke (I63) or any diagnosis of unspecified stroke (I64), transient ischaemic attack (G45), or peripheral emboli (174).

‡Arterial disease without previous myocardial infarction (I65, 170-73).

§Diagnostic codes used by the Swedish Board of Health of Welfare since 1987 to report alcohol related mortality (web appendix, table w1). 
Table 2| Atrial fibrillation related stroke risk in women compared with men

\begin{tabular}{lcc} 
Model & Hazard ratio (95\% Cl) & $\mathbf{P}$ \\
Univariable & $1.47(1.40$ to 1.54$)$ & $<0.001$ \\
\hline Adjusted for age & $1.16(1.11$ to 1.22$)$ & $<0.001$ \\
\hline Adjusted for $\mathrm{CHADS}_{2}$ score & $1.27(1.21$ to 1.33$)$ & $<0.001$ \\
\hline Multivariable (intention to treat) & $1.18(1.12$ to 1.24$)$ & $<0.001$ \\
\hline $\begin{array}{l}\text { Multivariable (intention to treat), age modelled with cubic } \\
\text { spline }\end{array}$ & $1.18(1.13$ to 1.25$)$ & $<0.001$ \\
\hline Multivariable with time dependent covariates* & $1.14(1.09$ to 1.19$)$ & $<0.001$ \\
\hline
\end{tabular}

Adjustments made for age, previous embolic event, heart failure, hypertension, diabetes, vascular disease, intracranial bleed, pacemaker, chronic obstructive pulmonary disease, pulmonary embolism, thyroid disease, platelet defect or coagulopathy, anaemia, renal failure, liver disease, alcohol index, dementia, frequent falls, cancer within three years, years since first diagnosis of atrial fibrillation, use of acetylsalicylic acid, clopidogrel, $\beta$ blockers, class I anti-arrhythmic drugs, class 3 anti-arrhythmic drugs, verapamil or diltiazem, dihydropyridine calcium blocker, digoxin, diuretics, angiotensin converting enzyme inhibitors and angiotensin receptor blockers, and statin.

*Warfarin was also included. 


\section{Figures}

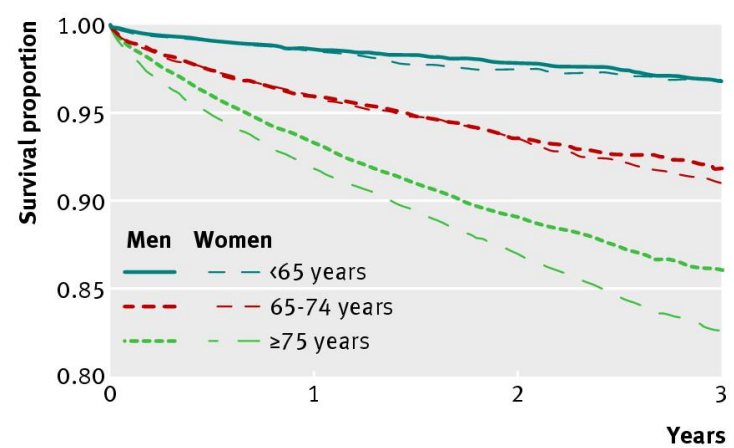

Fig 1 Unadjusted incidence of stroke in relation to age. Cox regression with main and interaction terms for sex and age group

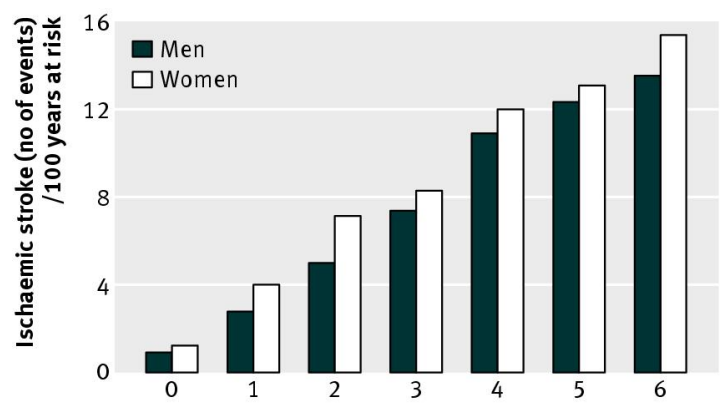

\begin{tabular}{|c|c|c|c|c|c|c|c|}
\hline \multicolumn{4}{|c|}{ Men with stroke } & & & \\
\hline Years at risk & 150 & 527 & 607 & 716 & 616 & 276 & 65 \\
\hline & 17533 & 19076 & 12138 & 9665 & 5628 & 2219 & 480 \\
\hline Women with s & stroke & & & & & & \\
\hline & 117 & 684 & 1021 & 1001 & 924 & 411 & 106 \\
\hline
\end{tabular}

Fig 2 Stroke rate in relation to $\mathrm{CHADS}_{2}$ score

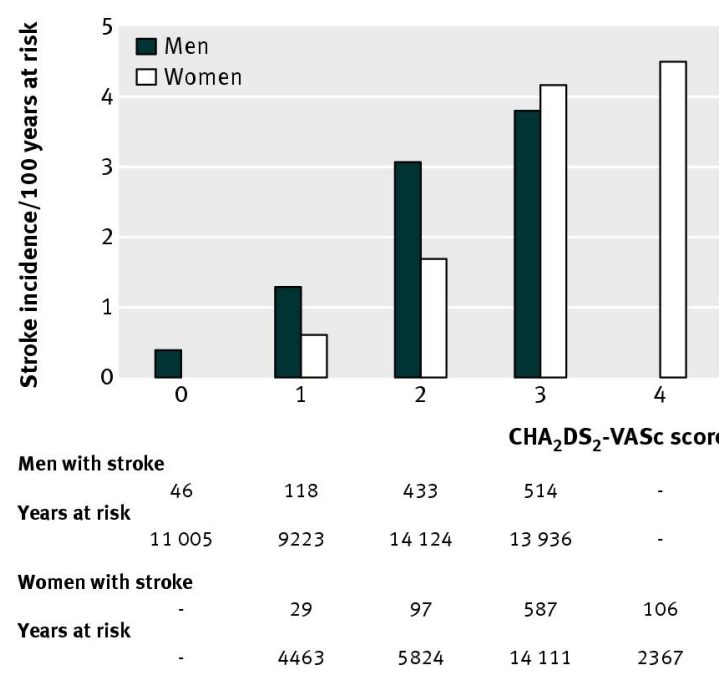

Fig 3 Stroke rate in patients with $\mathrm{CHADS}_{2}$ score $0-1$ in relation to $\mathrm{CHA}_{2} \mathrm{DS}_{2}-\mathrm{VASc}$ score 


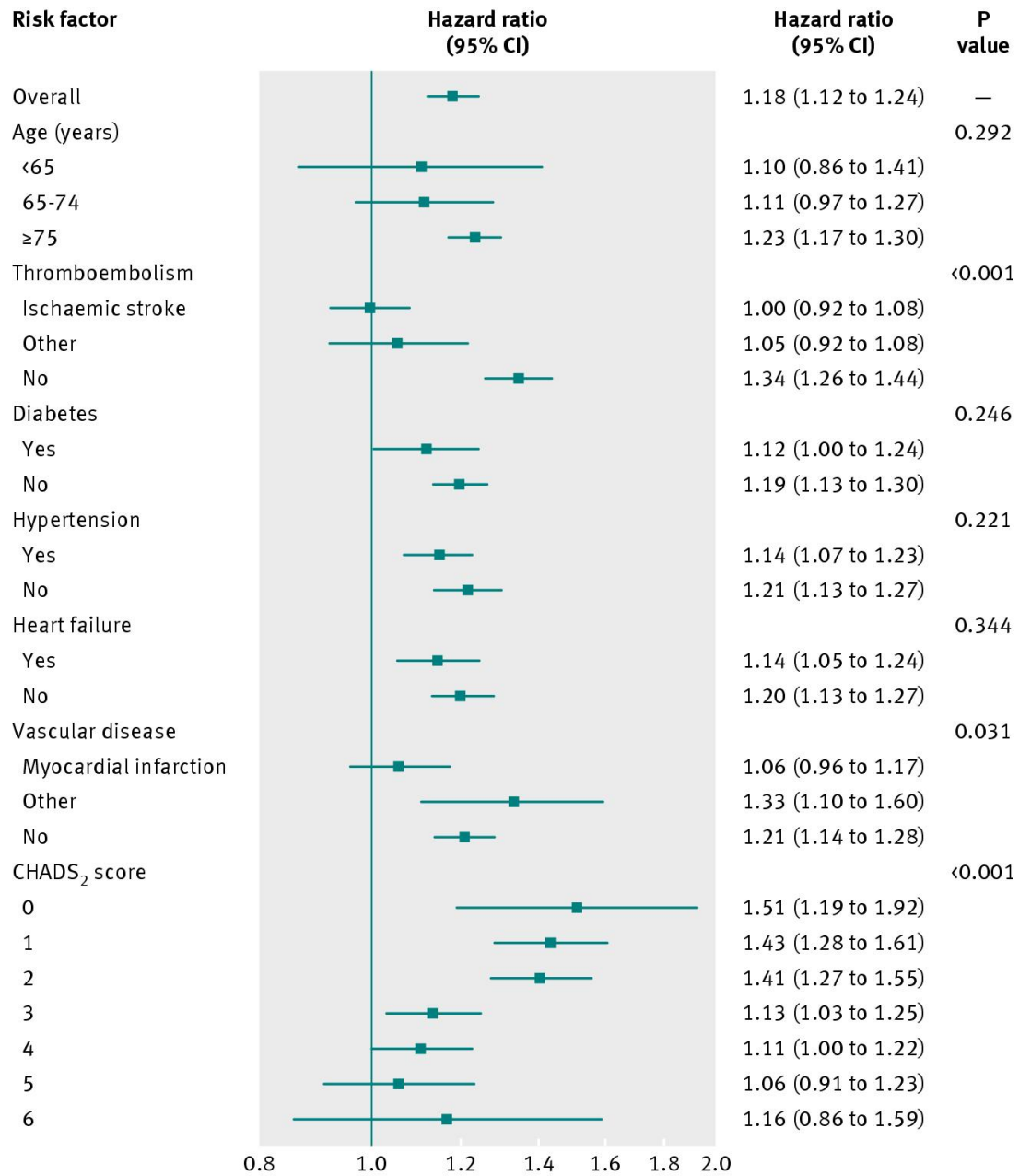

Fig 4 Risk of ischaemic stroke in women compared with men in relation to risk factors in $\mathrm{CHADS}_{2}$ and $\mathrm{CHA}_{2} \mathrm{DS}_{2}-\mathrm{VASc}$ schemes. All interactions modelled within the multivariable primary Cox regression, except $\mathrm{CHADS}_{2}$ (in which only sex and $\mathrm{CHADS}_{2}$ were included in the model). Interaction between sex and age was significant when age was included as a continuous variable in the model 